\title{
Characteristics and risk factors of Plasmodium falciparum malaria in Eastern and Central Nepal
}

\author{
Banjara MR ${ }^{1}$, Sirawaraporn $W^{2}$, Petmitr $\mathbf{S}^{1}$, Imwong $\mathbf{M}^{1}$, Joshi AB ${ }^{3}$, Chavalitshewinkoon-Petmitr $\mathbf{P}^{1}$ \\ ${ }^{1}$ Faculty of Tropical Medicine, Mahidol University, Bangkok, Thailand, ${ }^{2}$ Faculty of Science, Mahidol University, \\ Bangkok, Thailand, ${ }^{3}$ Institute of Medicine, Tribhuvan University, Kathmandu, Nepal
}

\begin{abstract}
Background: Very limited information is available on epidemiology of falciparum malaria in Nepal. Such information is very important for malaria control programmes. It is believed that malaria in Eastern region is imported from border districts of India and local transmission follows whereas it is indigenous in Central region. Therefore, the characteristics and risk factors of malaria are believed to be different in Eastern and Central Nepal.

Objective: The objective of the study is to describe and compare the characteristics and risk factors of falciparum malaria in Eastern and Central Nepal.

Materials and methods: This cross-sectional study was conducted in falciparum malaria endemic districts of Eastern and Central Nepal, during the period 2007 to 2008. We identified and collected information from 106 patients (62 from Eastern and 44 from Central region). Patient examination, clinical and laboratory assessment were done and patients were interviewed using structured questionnaire for malaria related characteristics, risk factors and behaviours.

Results: There were significant differences in risk factors and characteristics of falciparum malaria in the Central than the Eastern region. In the Central region, male, illiteracy and thatched roof hut were significant risk factors of falciparum malaria patients as compared to the Eastern region. Visits outside within one months, previous malaria within three months, taking antimalarial before confirmatory diagnosis were significantly higher in patients of the Eastern region as compared to the Central region.

Conclusion: Falciparum malaria in Nepal should not be seen as similar entity, and different strategies for prevention and control is needed for its diverse characteristics and endemicity.
\end{abstract}

Key words: Eastern and Central Nepal, falciparum malaria, risk factors

\begin{abstract}
$\mathrm{A}$ bout 70 percent of population in Nepal live in areas with unstable malaria transmission ${ }^{1}$. This includes Terai, the southern districts of Nepal bordering to India, where major malaria transmission was reported ${ }^{2}$. During the past few years, the cases caused by Plasmodium falciparum have increased. Approximately $80 \%$ of the total cases of all malaria and more than $90 \%$ of those of falciparum malaria occur in 12 districts ${ }^{1}$. It is noteworthy that variation of epidemiology of malaria occurs between different regions ${ }^{3}$ and over the time ${ }^{4}$ and these should be taken into consideration in malaria control programmes.
\end{abstract}

The trend of malaria incidence and epidemiology in recent years has been changing ${ }^{5}$. Malaria transmission in Nepal is focal and seasonal. Outbreaks of $P$. falciparum have been experienced in the past few years and the outbreak in October 2006 in Banke district claimed 33 lives. Late reporting, delay diagnosis and response were responsible for high case fatality. The outbreak was controlled after a month; however, details of the outbreak had not been studied. Updated information is available regarding the malaria epidemiology from various endemic countries; however, very limited information on malaria epidemiology is available in Nepal. Therefore, this study was conducted in Jhapa, Morang districts of the Eastern region and Dhanusha of the Central region. It is believed that malaria in the Eastern region is imported from border districts of India and local transmission occurs where as it is indigenous in the Central region. Therefore, in this study we compared and presented the characteristics and risk factors of falciparum malaria in the Eastern and the Central Terai of Nepal.

\footnotetext{
Correspondence

Assoc. Prof. Porntip Chavalitshewinkoon-Petmitr, PhD

Department of Protozoology, Faculty of Tropical Medicine

Mahidol University

Bangkok, Thailand

E-mail: tmppm@mahidol.ac.th
} 


\section{Materials and methods}

\section{Study design}

This was a descriptive cross-sectional study conducted during 2007 to 2008 in Jhapa, Morang and Dhanusha, the three important falciparum malaria endemic districts in the Eastern and Central regions of Nepal. Comparison of risk factors of falciparum malaria was made between Eastern and Central regions of Nepal.

\section{Study site}

The majority of malaria transmission in Nepal occurs in 12 priority districts. In Jhapa, falciparum malaria is endemic and accounts for almost fifty percent of the total national malaria cases. Both Jhapa and Morang are located in the Eastern region of Nepal and border with India, while Dhanusha is in the Central Nepal. Jhapa and Morang are moderate endemic districts whereas Dhanusha is low endemic district for malaria.

\section{Study patients and procedure}

The information was collected from falciparum malaria patients attending in selected health facilities of the study districts on their own initiative. The health facilities included Zonal Hospital, Primary Health Care Centre (PHCC), Health Posts (HP), sub-Health Post, and private diagnostic centres for malaria. The present study included 106 cases with P. falciparum - 62 from Eastern region, and 44 from Central region. Finger prick blood smear was prepared from patient who was reported to have fever with chills and suspected with malaria. Blood smear was stained and examined microscopically for falciparum malaria. Haemoglobin, body temperature, blood pressure were also measured. From microscopy confirmed falciparum malaria patients, clinical and epidemiological data such as age, gender, past history of malaria within three months, migration history, malaria risk related factors were obtained from a specific interview conducted by the physicians using standard structured questionnaire. The protocol of this study was reviewed and approved by the Institutional Review Board of the Institute of Medicine, Tribhuvan University, Nepal and the Faculty of Tropical Medicine, Mahidol University, Thailand. Written consent was obtained from each patient before drawing blood and taking interview.

\section{Data management and analysis}

Data was entered and analysed using SPSS 13.0. Percentages, mean, 95\% confidence intervals (CI) were calculated. t-test, $\chi 2$ test, and Fisher Exact test were used for statistical comparison between patient characteristics of Central and Eastern region of Nepal and $5 \%$ was used as a level of significance.

\section{Results}

Among the total patients, $8.9 \%$ were children and about $90 \%$ were young and adults male. The ratio of male to female was 5.2:1. The results showed that more males were infected in the Central region as compared to the Eastern region of Nepal $(\mathrm{p}=0.031)$. Proportion of mixed infection is $10.4 \%$ ( $P$. falciparum and $P$. vivax) and it is about double in the Eastern than the Central region. Regarding the risk factor of malaria, 67 (63.2\%) had thatched roof hut, $26(24.5 \%)$ had forest near the house, and $19(17.9 \%)$ had water logging near the house. (Table 1)

Patients visiting outside of living place within one month were $23.6 \%$ and among them $22.6 \%$ had visited different places of India. Previous malaria and fever within three months were found in 19.8\% and $11.3 \%$ among the total patients respectively. The occurrence of malaria previously within three months were significantly higher in Eastern region (32.3\%) than Central region $(2.3 \%) \quad(p<0.001)$. However, significantly more fever cases were reported in Central region $(22.7 \%)$ as compared to Eastern region (3.2\%) $(p=0.005)$. Mean number of days of illness before coming to health facility among total patients was 10.5 (95\% CI= 7.5-13.6). The delay was more in Central region than Eastern region; however, it was statistically insignificant ( $p=0.765$ ). Delay for consulting treatment more than 7 days was in $33.0 \%$ of patients, more patients in Central region as compared to Eastern region and it was statistically highly significant $(\mathrm{p}=$ 0.007). Likewise, $36.8 \%$ of the total patients consulted elsewhere and other health facilities before diagnosing as malaria patient. After becoming ill, $23.6 \%$ of total patients had taken antimalarial drug before coming and diagnosing as falciparum malaria patient in those health facilities. This was found higher in the Eastern region $(30.6 \%)$ as compared to the Central region (13.6\%) and was statistically significant $(p=0.042)$. (Table 2) 
Table 1: Demographic and other malaria related characteristics of the falciparum malaria patients in Eastern and Central Nepal

\begin{tabular}{|c|c|c|c|}
\hline Variables & $\begin{array}{c}\text { Eastern region } \\
(n=62)\end{array}$ & $\begin{array}{c}\text { Central region } \\
(n=44)\end{array}$ & p-value \\
\hline Patient less than or equal to 15 years & $8(12.9 \%)$ & $4(9.1 \%)$ & $0.569 *$ \\
\hline Mean age of the patients in years & $30.1 \pm 14.1$ & $32.1 \pm 11.7$ & $0.435 * *$ \\
\hline Male to female ratio & $3.4: 1$ & $13.6: 1$ & $0.029 * * *$ \\
\hline Illiterate patients & $20(32.3 \%)$ & $25(56.8 \%)$ & $0.008^{*}$ \\
\hline Patients with agriculture occupation & $46(74.2 \%)$ & $39(88.6 \%)$ & $0.490^{*}$ \\
\hline Patients with daily labour & $7(11.3 \%)$ & $2(4.5 \%)$ & $0.129 * * *$ \\
\hline Patients with joint family & $36(58.1 \%)$ & $12(27.3 \%)$ & $0.002 *$ \\
\hline Patients having long lasting insecticide treated nets in the house & $2(3.2 \%)$ & $6(13.6 \%)$ & $0.046^{* * *}$ \\
\hline Patients having forest near the house & $14(22.6 \%)$ & $12(27.3 \%)$ & $0.580^{*}$ \\
\hline Patients having water logging near the house & $14(22.6 \%)$ & $5(11.4 \%)$ & $0.138 *$ \\
\hline Patients having thatched roof hut & $30(48.4 \%)$ & $37(84.1 \%)$ & $<0.001 *$ \\
\hline
\end{tabular}

*Chi-square test, $* *$ Independent sample t-test, $* * *$ Fisher exact test

Table 2: Comparison of risk factors of falciparum malaria

\begin{tabular}{|l|l|l|l|}
\hline Variables & Eastern region & Central region & p-value \\
\hline Patients visiting outside living place within one month & $24(38.7 \%)$ & $1(2.3 \%)$ & $<0.001 * * *$ \\
\hline Patients visiting different places of India & $23(37.1 \%)$ & $1(2.3 \%)$ & - \\
\hline Patients with malaria disease within three months & $20(32.3 \%)$ & $1(2.3 \%)$ & $<0.001 * * *$ \\
\hline Patients with non-malarial fever within three months & $2(3.2 \%)$ & $10(22.7 \%)$ & $0.005^{* * *}$ \\
\hline $\begin{array}{l}\text { Mean number of days of illness before coming to health facility } \\
(95 \% \mathrm{CI})\end{array}$ & $\begin{array}{l}10.2 \pm 18.7 \\
(5.4-14.9)\end{array}$ & $\begin{array}{l}11.1 \pm 11.3 \\
(7.6-14.5)\end{array}$ & $0.765^{* *}$ \\
\hline $\begin{array}{l}\text { Consulted elsewhere before diagnosing as malaria patient in the } \\
\text { health facility }\end{array}$ & $24(38.7 \%)$ & $15(34.1 \%)$ & $0.627 *$ \\
\hline Patients taking antimalarial before coming to the health facility & $19(30.6 \%)$ & $6(13.6 \%)$ & $0.042^{*}$ \\
\hline
\end{tabular}

*Chi-square test, **Independent sample t-test, ***Fisher exact test

\section{Discussion}

Updated information on epidemiology of the disease is essential for the development of effective control programmes. In the case of Nepal, epidemiology of vivax as well as falciparum malaria has never been described and published. In the present study, we collected information from limited number of patients in two years, and carried out an epidemiological study comparing falciparum malaria in patients living in the Eastern and Central region of Nepal.

Ten out of eleven member countries of WHO Southeast Asia region are malaria endemic and accounts for 8.5 percent of the global morbidity. In the region, $95 \%$ cases and deaths are in India, Indonesia, Myanmar and Bangladesh $^{6}$. Nepal is low endemic country with high incidence of vivax malaria. However, in recent years, the cases of falciparum malaria are increasing in this mountainous country in low land Terai. This may be due to climate change and its impact on vector borne diseases ${ }^{7,8}$. The reversing trend of vivax and falciparum malaria in Nepal is reported over the years. Increased precipitation and temperature are reported significant for the increased number of malaria cases in $\mathrm{Nepal}^{9}$. Besides, increased proportion of falciparum malaria could be due to better diagnosis of the cases. Very limited information on mixed infection of $P$. falciparum and $P$. vivax is available and molecular analysis will be important to detect the proportion of mixed infection.

In our study, it appears that most of the cases were adult males, suggesting preferential infection of falciparum malaria in mobile and economically active population. In population who live in high transmission area and stably exposed to malaria, the prevalence and density of parasitaemia are high in younger age groups ${ }^{10,11}$, whereas in low transmission area all populations especially mobile are affected. Based on age distribution of falciparum malaria, Nepal is categorised as low transmission area for falciparum malaria. In most high malaria transmission countries, the ratio of male to 
female patients with malaria infection is nearly equal. However, our data showed that the number of males with $P$. falciparum infection was 5 times higher than the number of females with P. falciparum. In addition, the ratio was significantly higher in the Central region compared to the Eastern region of Nepal. Since females do not go far for work, they are less likely to be infected with malaria. Poor utilisation of the health services by females may be another reason since this study is based on health services. Socio-demographic characteristics also indicate that the incidence of falciparum malaria is more among the people with agriculture and those whose occupations are daily labours. Falciparum malaria is significantly higher in people living in the Central region with agriculture occupation compared to people in the Eastern region, indicating that falciparum malaria is common in labour migrants in the Eastern region of the country.

In Nepal, the malaria cases are both indigenous and imported. Our data reveal that about one fourth of the falciparum malaria infected patients visited different regions of India. The number of cases is much higher in the Eastern region compared to those in the Central region, suggesting that falciparum malaria in the Eastern region might be from both indigenous and imported sources, whereas those found in the Central region of the country were indigenous. In the Eastern region of Nepal, many people go for work in India where malaria is endemic. Therefore, it is recommended that those who return from malaria endemic areas of India should be counselled at the quarantine check post and consult with health facilities immediately if they have fever with chills. This will help lower the transmission potential. In addition, immediate measures could be taken for effective falciparum malaria management, including malaria preventive travel advice given to visitors to India. The molecular analysis also revealed that malaria in Eastern Nepal is due to both importation and local transmission (data not shown).

Our data also reveal that about $20 \%$ of the patients previously had malaria within three months. This is significantly higher in Eastern region than Central region indicating that the endemicity of malaria is quite different in these regions. The patient delay for consulting health facilities was 10.5 days and delay more than 7 days was in almost 33 percent of the patients. The delay was more in Central region than Eastern region. Those who had visited other places before diagnosing as falciparum malaria, 23.6 percent took antimalarials which indicate that improper prescription of the drugs without confirmatory diagnosis. Almost one third patients of Eastern region took antimalarials which is significantly higher than those of Central region patients. Since the patients come with falciparum malaria, it is possible that they got wrong antimalarial or they were re-infected. This could contribute to antimalarial drug resistance and development of further complications in the patients. Our molecular study on drug resistance also reveals this and will be published somewhere. In Nepal, diagnosis method of the malaria parasite is not proper and most of the health workers use chloroquine which is already ineffective against $P$. falciparum, though the drug is still highly effective against $P$. vivax. Treatment failure with sulfadoxine-pyrimethamine combination for falciparum malaria in the Eastern Nepal has also been reported by some studies ${ }^{12,13}$. The artemisinin based combination therapy (ACT) has recently been started in Nepal. However, ineffective antimalarials are being used due to unavailability of ACT in the peripheral health facilities. The health workers need to be trained for the diagnosis and treatment of falciparum malaria since the cases of deadly malaria are increasing.

In summary, malaria cases with history of visit to other places specially malaria endemic areas of India, malaria cases with previous malaria within three months, were significantly higher in the Eastern than in the Central region. The malaria patients from the Eastern region took antimalarials before confirmatory diagnosis and it was significantly higher than those from the Central region. These evidences support that the falciparum malaria in the Eastern region of Nepal are mixed of imported and indigenous cases, whereas those in the Central region are mostly indigenous cases. Therefore, falciparum malaria in Nepal should not be seen as single entity, and different strategies for prevention and control may be designed for its diverse characteristics and endemicity.

\section{Conclusion}

The risk factors of falciparum malaria and patient characteristics in Eastern and Central region of Nepal are significantly different indicating that different strategies should be adopted for the prevention and control of falciparum malaria. Health education for timely consultation of the patient to the health facilities is important.

\section{Acknowledgements}

The authors would like acknowledge falciparum malaria patients involved in this study. This study was financially supported by UNDP/World Bank/WHO Special Programme for Research and Training in Tropical Diseases (TDR) Geneva, Switzerland for PhD study of MR Banjara. 


\section{References}

1. Department of Health Services, Ministry of Health, Nepal. Annual Report 2002/2003. Kathmandu: Department of Health Services; 2004.

2. World Health Organization, Regional Office for South-East Asia. Malaria Situation in SEAR Countries, Nepal 2006 [cited on December 2008]. Available at http://w3.whosea.org/en/ Section10/Section21/ Section340.htm.

3. Remme JH, Binka F, Nabarro D. Toward a framework and indicators for monitoring Roll Back Malaria. Am J Trop Med Hyg. 2001; 64 (Suppl 1-2): 76-84.

4. Ceesay SJ, Casals-Pascual C, Erskine J, Anya SE, Duah NO, Fulford AJ, et al. Changes in malaria indices between 1999 and 2007 in The Gambia: a retrospective analysis. Lancet. 2008, 372 (9649):1545-54.

5. World Health Organization. World Health Report 2005. Geneva: World Health Organization; 2005.

6. World Health Organization, Regional Office for South-East Asia. Malaria fact sheet 2009 [cited on December 2008]. Available at http://www. searo.who.int/LinkFiles/Malaria_FactsheetWMD2009.pdf.

7. Garg A, Dhiman RC, Bhattacharya S, Shukla PR. Development, malaria and adaptation to climate change: A case study from India. Environ Manage. 2009;43:779-89.
8. Zhang Y, Peng B, Hiller JE. Climate change and the transmission of vector-borne diseases: a review. Asia Pac J Public Health. 2008, 20 (1): 64-76.

9. Dahal S. Climatic determinants of malaria and kala-azar in Nepal. Regional Health Forum. 2008, 12 (1): 32-7.

10. Trape JF, RogierC.Combating malaria morbidity and mortality by reducing transmission. Parasitol Today. 1996; 12: 236-40.

11. Syafruddin D, Krisin K, Asih P, Sekartuti, Dewi RM, Coutrier F, et al. Seasonal prevalence of malaria in West Sumba district, Indonesia. Malaria Journal. 2009; 8: 8.

12. Thapa S, Hollander J, Linehan M, Cox-Singh $\mathrm{J}$, Bista MB, Thakur GD, Davis WA, et al. Comparison of artemether-lumefantrine with sulfadoxine-pyrimethamine for the treatment of uncomplicated falciparum malaria in Eastern Nepal. Am J Trop Med Hyg 2007; 77(3): 42330.

13. Wijeyaratne PM, Chand PB, Valech N, Shahi B, Adak T, Ansari MA, Jha J, et al. Therapeutic efficacy of antimalarial drugs along the Eastern Indo-Nepal border: a cross-border collaborative study. Trans R Soc Trop Med Hyg. 2005; 99: 423-9. 